\title{
Incentivizing Effort in Interdependent Security Games Using Resource Pooling*
}

\author{
Mohammad Mahdi Khalili \\ EECS, University of Michigan \\ khalili@umich.edu
}

\author{
Xueru Zhang \\ EECS, University of Michigan \\ xueru@umich.edu
}

\author{
Mingyan Liu \\ EECS, University of Michigan \\ mingyan@umich.edu
}

\begin{abstract}
We consider an InterDependent Security (IDS) game with networked agents and positive externality where each agent chooses an effort/investment level for securing itself. The agents are interdependent in that the state of security of one agent depends not only on its own investment but also on the other agents' effort/investment. Due to the positive externality, the agents under-invest in security which leads to an inefficient Nash equilibrium (NE). While much has been analyzed in the literature on the under-investment issue, in this study we take a different angle. Specifically, we consider the possibility of allowing agents to pool their resources, i.e., allowing agents to have the ability to both invest in themselves as well as in other agents. We show that the interaction of strategic and selfish agents under resource pooling (RP) improves the agents' effort/investment level as well as their utility as compared to a scenario without resource pooling. We show that the social welfare (total utility) at the NE of the game with resource pooling is higher than the maximum social welfare attainable in a game without resource pooling but by using an optimal incentive mechanism. Furthermore, we show that while voluntary participation in this latter scenario is not generally true, it is guaranteed under resource pooling.
\end{abstract}

\section{CCS CONCEPTS}

-Networks $\rightarrow$ Network economics; $\bullet$ Security and privacy $\rightarrow$ Network security.

\section{INTRODUCTION}

The increasing rate and scale of cyber crime is placing significant pressure on organizations to improve their security posture. At the same time, the interdependent nature of cyber risks means one's state of security is not just the result of one's own security practices and investments, but of others' connected to it, e.g., through attack propagation and supply chain relationships. Decision making in such a scenario has often been modeled as an InterDependent Security (IDS) game [18]. The most critical issue that arises in IDS games is free-riding where an entity under-invests in security and

\footnotetext{
${ }^{*}$ This work is supported by the NSF under grants CNS-1616575, CNS-1739517, and by the ARO under contract W911NF1810208.

Permission to make digital or hard copies of all or part of this work for personal or classroom use is granted without fee provided that copies are not made or distributed for profit or commercial advantage and that copies bear this notice and the full citation on the first page. Copyrights for components of this work owned by others than ACM must be honored. Abstracting with credit is permitted. To copy otherwise, or republish, to post on servers or to redistribute to lists, requires prior specific permission and/or a fee. Request permissions from permissions@acm.org.

NetEcon'19, fune 28, 2019, Phoenix, AZ, USA

(C) 2019 Association for Computing Machinery.

ACM ISBN 978-1-4503-6837-7/19/06...\$15.00

https://doi.org/10.1145/3338506.3340272
}

takes advantage of others' efforts. As a result, the Nash equilibrium (NE) in IDS games is inefficient and individuals' investment in security is below the optimum [14, 27].

To address the free-riding issue and incentivize individuals to improve their security investment, various mechanisms have been proposed. [7] shows that bonus and penalty based on agents' security outcome can improve network security. [13] and [15] show that cyber insurance in the presence of a quantitative security assessment (pre-screening) is able to improve the security investment and address the free-riding issue. Ioannidis et al. in [11] show that public coordination under the guidance of a well-informed steward can improve the resilience of the system to attacks. In [20], the Pivotal (VCG) and Externality mechanisms are analyzed (both are in the form of a taxation/subsidy mechanism) to induce socially optimal outcome in IDS games; however, it also shows that no tax mechanism can simultaneously satisfy both budget balance and voluntary participation constraints. This is because security is a non-excludable public good and individuals continue to benefit from other's effort even if they unilaterally opt out of the mechanism.

All of the above studies assume the existence of a central entity, a social planner, a coordinator, or a steward. In this study, different from existing literature, we shall take a different approach to inducing socially desirable or optimal outcomes in this type of IDS games. Specifically, we consider the absence of such a central entity, and instead model the presence of resource pooling (RP) by allowing agents to have the ability to both invest in themselves as well as in other agents, so that they can choose to not only improve its own but also others' state of security. This modeling choice leads to a different IDS game, referred to as the RP-augmented IDS game, or simply RP-IDS below. In practice, exerting efforts on other agents' behalf has context dependent interpretations, such as providing product/service discounts to customers by a service provider, as well as funding open source development. Note that both IDS game and RP-IDS are non-cooperative games where agents selfishly choose their actions to maximize their own utility. Thus our model is different from that of a cooperative game [24-26] where players form coalitions and choose actions to maximize the utility of the coalition they belong to. A cooperative game can lead to improved network security as compared to a non-cooperative one if the cost of forming coalitions is sufficiently low, but forming coalition is not always possible due to cultural, economical, or social reasons [22].

Specifically, we study the IDS game with a weighted total effort and quadratic cost model under two scenarios: (i) no RP (the original IDS game), where each agent exerts effort only to improve his own security; and (ii) with RP (RP-IDS), where selfish agents pool their resources. Our main findings are summarized as follows. 
1) Both games have a unique NE. At the NE of the RP-IDS game, every agent obtains higher utility as compared to that under the NE of the IDS game.

2) The social welfare (measured by total utility) at the NE of the RP-IDS game is higher than that under the socially optimal outcome of the IDS game, induced by mechanisms such as VCG and externality mechanisms [20]. In other words, as a mechanism, $\mathrm{RP}$ outperforms these tax-based mechanisms.

3) While the VCG and externality mechanisms cannot guarantee voluntary participation while imposing budget balance [20], we show that in the RP-IDS game no agent will unilaterally opt out of resource pooling (while continue to be part of the IDS game), thereby ensuring voluntary participation.

\section{Related Works}

Distributed mechanism framework has been proposed to induce socially optimal outcome in a distributed manner, i.e., message transmission is performed locally, and mechanism/tax functions depend on messages from neighboring agents [8, 9, 23]. Even though distributed mechanisms are viable options to implement the socially optimal outcome without a central planner, they still cannot be used in IDS games because they are in the form of taxation mechanism and not able to satisfy the notion of voluntary participation [20].

Outside the incentive context, IDS games have been extensively studied in the literature $[2,10,12,17,19]$; we reference some of the more relevant ones below. Ann Miura-Ko et al. [19] consider a linear influence network and find a condition on the dependence matrix to guarantee the existence and uniqueness of the NE. Hota and Sundaram in [10] consider IDS games under behavioral probability weighting and show that security risk can be reduced by such weighting strategies. [2] shows that the under-investment issue similarly exists in a two-stage game model. [17] examines the relationship between risk exposure and agents' degrees in the dependence graph.

The most related work to the present paper is [16] which studies the role of resource pooling in public good provision games with limited resources. The main conclusion of [16] is that resource pooling under limited resources is not able to induce socially optimal outcomes, for which an incentive mechanism is needed.

In the remainder of the paper, we present the IDS game model without RP, and the RP-IDS game model, and their associated analysis, in Sec. 2 and 3, respectively. A number of discussions are given in Sec. 4. Sec. 5 concludes the paper.

\section{INTERDEPENDENT SECURITY GAME WITHOUT RESOURCE POOLING (IDS)}

Consider $n$ agents on a directed, weighted graph denoted by $\mathcal{G}=$ $(\mathcal{V}, \mathcal{E}, X)$, where $\mathcal{V}=\{1,2, \cdots, n\}$ is the set of $n$ agents, $\mathcal{E} \subseteq$ $\{(i, j) \mid i \neq j, i, j \in \mathcal{V}\}$ the set of edges between them, and $X=$ $\left[x_{i j}\right]_{n \times n}$ the adjacency weight matrix of this graph, where $x_{i j}>$ $0,(i, j) \in \mathcal{E}$ is the edge weight, $x_{i j}=0,(i, j) \notin \mathcal{E}$, and $x_{i i}=0, i \in \mathcal{V}$. An edge $(i, j) \in \mathcal{E}$ indicates that agent $i$ depends on agent $j$ (or agent $j$ influences $i$ ) with the degree of dependence given by edge weight $x_{i j}$. Dependence need not be symmetrical, i.e., $x_{i j} \neq x_{j i}$ in general. We assume $x_{i i}=0, i \in \mathcal{V}$. Agent $i$ exerts effort $e_{i} \geq 0$ towards securing himself, incurring cost $b_{i} \cdot e_{i}^{2}\left(b_{i}>0\right.$ a constant). Given effort profile $\boldsymbol{e}=\left[e_{1}, e_{2}, \cdots, e_{n}\right]^{T}$, agent $i$ has utility

$$
u_{i}\left(e_{i}, e_{-i}\right)=-l_{i}+a_{i} \cdot e_{i}+e_{i} \cdot\left(\sum_{j=1}^{n} x_{i j} e_{j}\right)-b_{i} \cdot e_{i}^{2},
$$

where $e_{-i}$ denotes all elements in $\boldsymbol{e}$ excluding $e_{i},-l_{i}$ a nominal loss agent $i$ suffers without any effort, $a_{i} \cdot e_{i}, a_{i} \geq 0$, the benefit it derives from effort $e_{i}$, and $e_{i} \cdot x_{i j} \cdot e_{j}$ the benefit it derives from other agents' efforts. This term suggests that an agent who does not exert effort also does not benefit from other's efforts. This may be interpreted as implying that the type of security products or technologies agents use are complementary. Note that $x_{i j} \geq 0$ indicates a case of positive externality between agents $i$ and $j$; see e.g., [6] for IDS games with negative externalities. This is a form of the quadratic utility function widely used in the literature of network games [3, 5] and IDS games [4, 21]; it can be viewed as a second-order approximation of any utility function.

The interaction of agents induces a game, denoted as $G=\left\{\mathcal{V},\left\{u_{i}(.)\right\}_{i \in \mathcal{V}}, A=[0,+\infty)^{n}\right\}$, where $A$ is the action space. In the rest of the paper, we shall use the terms exerted effort, actions and security investments interchangeably. For convenience of notation, when comparing two games given by the same $\mathcal{V}, \mathcal{E}$ but different weight matrices $X_{1}$ and $X_{2}$, we will denote the resulting games as $G\left(X_{1}\right)$ and $G\left(X_{2}\right)$, respectively. Next we analyze the equilibrium of game $G$.

\subsection{Equilibrium Analysis}

Let $B r_{i}\left(e_{-i}\right)$ denote the best response function of agent $i$. Using the first order condition we have

$$
\begin{aligned}
B r_{i}\left(e_{-i}\right) & =\arg \max _{e \geq 0} u_{i}\left(e, e_{-i}\right) \\
& =\frac{a_{i}}{2 b_{i}}+\frac{1}{2 b_{i}} \sum_{j=1}^{n} x_{i j} e_{j} .
\end{aligned}
$$

We will primarily focus on pure strategy Nash equilibrium (NE), and for simplicity of exposition for the rest of the paper Nash equilibrium refers to a pure strategy NE. An NE is the fixed point of the best response mapping. Let $\hat{\boldsymbol{e}}$ denote the agents' effort at the NE of game $G$; then $\hat{\boldsymbol{e}}$ satisfies the following equations:

$$
\begin{aligned}
2 b_{i} \hat{e}_{i}-\sum_{j=1}^{n} x_{i j} \hat{e}_{j} & =a_{i}, \quad i=1,2 \cdots, n \\
\text { or }(2 \cdot B-X) \cdot \hat{\boldsymbol{e}} & =\boldsymbol{a},
\end{aligned}
$$

where $B$ is a matrix with $b_{i}$ 's on its main diagonal and zeros everywhere else, and $\boldsymbol{a}=\left[a_{1}, a_{2}, \cdots, a_{n}\right]^{T}$.

We make the following assumption on cost $b_{i}$ to ensure that the effort levels are bounded at the NE. More discussion on this assumption is provided in Section 4.1.

Assumption 1. $2 b_{i} \geq \sum_{j=1}^{n} x_{i j}, \forall i \in \mathcal{V}$.

Under this assumption, we have the following lemma on the best response mapping and the NE of game $G$.

Theorem 2.1. Under Assumption 1, matrix $(2 B-X)$ is invertible and $\hat{\boldsymbol{e}}=(2 \cdot B-X)^{-1} \cdot \boldsymbol{a}$ is the unique NE of game $G$.

Proof. See Appendix. 
Note that Theorem 2.1 holds for any non-negative vector $\boldsymbol{a}$, which leads to the following corollary.

COROLlary 2.2. Under Assumption 1, all entries of matrix $(2 \cdot B-$ $X)^{-1}$ are non-negative. Furthermore, let $X$ and $\tilde{X}$ be two adjacency matrices over the same $\mathcal{V}$ and $\mathcal{E}$. Consider the games $G(X)$ and $G(X+\tilde{X})$, and their respective $N E \hat{\boldsymbol{e}}$ and $\tilde{\boldsymbol{e}}$. If $2 b_{i} \geq \sum_{j=1}^{n}\left[x_{i j}+\tilde{x}_{i j}\right]$, then $\tilde{\boldsymbol{e}} \geq \hat{\boldsymbol{e}} .^{1}$ In other words, agents exert higher effort at the NE given stronger externality.

Proof. Let $\mathbf{0} \in \mathbb{R}^{n}$ be a zero vector. By Theorem 2.1, we know that $(2 \cdot B-X)^{-1} \cdot \tilde{\boldsymbol{a}} \geq \mathbf{0}$ for any non-negative vector $\tilde{\boldsymbol{a}}$. Set $\tilde{a}_{i}=1$ and $\tilde{a}_{j}=0, \forall j \neq i$ and $\tilde{\boldsymbol{a}}=\left[\tilde{a}_{1}, \cdots, \tilde{a}_{n}\right]^{T}$. Then, $(2 \cdot B-X)^{-1} \cdot \tilde{\boldsymbol{a}} \geq \mathbf{0}$ is the $i$ th column of $(2 \cdot B-X)^{-1}$. Because $i$ is arbitrary, all columns of $(2 \cdot B-X)^{-1}$ are non-negative. Moreover, we have,

$$
\begin{aligned}
(2 B-X) \cdot \hat{\boldsymbol{e}} & =\boldsymbol{a} \\
(2 B-X-\tilde{X}) \cdot \tilde{\boldsymbol{e}} & =\boldsymbol{a} \Longrightarrow \\
\tilde{\boldsymbol{e}}=(2 B-X)^{-1} \cdot \boldsymbol{a} & +(2 B-X)^{-1} \cdot \tilde{X} \cdot \tilde{\boldsymbol{e}} \\
& =\hat{\boldsymbol{e}}+\underbrace{(2 B-X)^{-1} \cdot \tilde{X} \cdot \tilde{\boldsymbol{e}} \geq \hat{\boldsymbol{e}}}_{\geq \mathbf{0}}
\end{aligned}
$$

\subsection{Socially Optimal Outcome}

We now consider the socially optimal effort levels for the IDS game. Denote by $\boldsymbol{e}^{*}=\left[e_{1}^{*}, e_{2}^{*}, \cdots, e_{n}^{*}\right]$, the socially optimal effort profile maximizes the total utility:

$$
\boldsymbol{e}^{*} \in \arg \max _{\boldsymbol{e} \in A} \sum_{i=1}^{n} u_{i}\left(e_{i}, e_{-i}\right)
$$

To ensure the existence of a socially optimal strategy, we make the following assumption (see Section 4.1 for more discussion).

Assumption 2. $2 b_{i} \geq \sum_{j=1}^{n}\left[x_{i j}+x_{j i}\right], \forall i \in \mathcal{V}$.

Theorem 2.3. Let $\hat{\boldsymbol{e}}$ be the effort level at the NE of game $G$ and $\boldsymbol{e}^{*}$ be the socially optimal effort level. Under Assumption 2 we have:

(1) $\boldsymbol{e}^{*}=\left(2 B-X-X^{T}\right)^{-1} \cdot \boldsymbol{a}$;

(2) $e_{i}^{*} \geq \hat{e}_{i}, \forall i$.

That is, every agent exerts higher effort at the socially optimal solution compared to the NE.

Proof. See Appendix.

Remark: The above shows that the socially optimal effort profile of game $G(X)$, given by $\boldsymbol{e}^{*}=\left(2 B-X-X^{T}\right)^{-1} \cdot \boldsymbol{a}$, also happens to be the NE of game $G\left(X+X^{T}\right)$. Also note that for game $G(X)$, while the total utility under $\boldsymbol{e}^{*}$ is higher than that under the NE $\hat{\boldsymbol{e}}$, this may or may not be true for agents' individual utility, as the following example shows.

\footnotetext{
$\left.\overline{{ }^{1} \boldsymbol{v}=\left[v_{1} \cdots v_{n}\right.}\right]^{T} \geq \boldsymbol{\theta}=\left[\theta_{1} \cdots \theta_{n}\right]^{T}$ means that $v_{i} \geq \theta_{i}, \forall i$
}

Example 2.4. Consider the following IDS game:

$$
\begin{aligned}
n & =2, b_{1}=b_{2}=1, a_{1}=a_{2}=1 \\
x_{12} & =0.1, x_{21}=0.9, l_{1}=l_{2}=1 \\
\hat{\boldsymbol{e}} & =(2 B-X)^{-1} \cdot \boldsymbol{a}=[0.53710 .7417]^{T} \\
u_{1}(\hat{\boldsymbol{e}}) & =-0.7115, u_{2}(\hat{\boldsymbol{e}})=-0.4499 \\
\boldsymbol{e}^{*} & =\left(2 B-X-X^{T}\right)^{-1} \cdot \boldsymbol{a}=\left[\begin{array}{ll}
1 & 1
\end{array}\right]^{T} \\
u_{1}\left(\boldsymbol{e}^{*}\right) & =-0.9000, u_{2}\left(\boldsymbol{e}^{*}\right)=-0.1000
\end{aligned}
$$

In this example, agent 1 has higher influence on agent $2\left(x_{21}>x_{12}\right)$; agent 2 benefits from socially optimal effort $\left(u_{2}\left(\boldsymbol{e}^{*}\right)>u_{2}(\hat{\boldsymbol{e}})\right)$, while agent 1 's utility worsens even though it exerts higher effort under $e^{*}$.

Example 2.5. Consider the following IDS game where both agents benefit from the socially optimal outcome:

$$
\begin{aligned}
n & =2, b_{1}=b_{2}=1, a_{1}=a_{2}=1 \\
x_{12} & =x_{21}=0.5, l_{1}=l_{2}=1 \\
\hat{\boldsymbol{e}} & =(2 B-X)^{-1} \cdot \boldsymbol{a}=\left[\frac{2}{3}, \frac{2}{3}\right]^{T} \\
u_{1}(\hat{\boldsymbol{e}}) & =u_{2}(\hat{\boldsymbol{e}})=-\frac{5}{9}=-0.5555 \\
\boldsymbol{e}^{*} & =\left(2 B-X-X^{T}\right)^{-1} \cdot \boldsymbol{a}=\left[\begin{array}{ll}
1 & 1
\end{array}\right]^{T} \\
u_{1}\left(\boldsymbol{e}^{*}\right) & =u_{2}\left(\boldsymbol{e}^{*}\right)=-0.5
\end{aligned}
$$

These examples show that socially optimal outcome is not necessarily desirable to all agents. Mechanism design in the context of IDS games aims to incentivize agents to exert higher effort than that under the NE. In the next section, we will examine the impact of introducing resource pooling as a mechanism to improve agents' effort and social welfare.

\section{INTERDEPENDENT SECURITY GAME WITH RESOURCE POOLING (RP-IDS)}

Consider the same IDS game setting. Let $\boldsymbol{e}_{i}=\left[e_{i 1}, e_{i 2}, \cdots, e_{i n}\right]^{T}$ be the action of agent $i$ where $e_{i j} \geq 0$ denotes the effort exerted by agent $i$ on behalf of agent $j$. Moreover, agent $i$ incurs cost $b_{j} \cdot e_{i j}^{2}$ by exerting effort $e_{i j}$ on behalf of agent $j$, i.e., the cost of exerting an effort on behalf of agent $j$ depends on $j .{ }^{2}$ Let $E=\left[\boldsymbol{e}_{1}, \boldsymbol{e}_{2}, \cdots, \boldsymbol{e}_{n}\right]^{T}$ be an $n \times n$ matrix that denotes the effort profile, and let $E_{i}=$ $\sum_{j=1}^{n} e_{j i}$ denote the total effort exerted on behalf of agent $i$. Agent $i$ 's utility given profile $E$ is:

$$
\begin{aligned}
v_{i}\left(\boldsymbol{e}_{i}, \boldsymbol{e}_{-i}\right) & =-l_{i}+a_{i}\left(\sum_{j=1}^{n} e_{j i}\right)-\sum_{k=1}^{n} b_{k} \cdot e_{i k}^{2} \\
& +\left(\sum_{j=1}^{n} e_{j i}\right) \cdot \sum_{k=1}^{n} x_{i k} \cdot\left(\sum_{r=1}^{n} e_{r k}\right) \\
& =-l_{i}+a_{i} E_{i}+E_{i} \cdot \sum_{j=1}^{n} x_{i j} E_{j}-\sum_{k=1}^{n} b_{k} \cdot e_{i k}^{2} .
\end{aligned}
$$

The interaction of agents induces the RP-IDS game $G_{r p}=\left\{\mathcal{V},\left\{v_{i}\right\}_{i \in \mathcal{V}}, A_{r p}=[0,+\infty)^{n^{2}}\right\}$, where $A_{r p}$ is the action

\footnotetext{
${ }^{2}$ An example of this is one firm providing security training for employees of another firm; the incurred training cost depends on the trainees' education level.
} 
space under resource pooling. By first order condition the best response function of agent $i$ satisfies the following:

$$
\begin{aligned}
\boldsymbol{e}_{i} & =B r_{i}\left(\boldsymbol{e}_{-i}\right) \\
e_{i i} & =\frac{a_{i}}{2 b_{i}}+\frac{\sum_{k=1}^{n} x_{i k} \cdot E_{k}}{2 b_{i}} \\
e_{i j} & =\frac{x_{i j} \cdot E_{i}}{2 b_{j}}, \forall j \neq i
\end{aligned}
$$

Let $\hat{E}=\left[\hat{e}_{i j}\right]_{n \times n}$ be the NE of game $G_{r p}$ and $\hat{E}_{i}=\sum_{j=1}^{n} \hat{e}_{j i}$ the total effort exerted on behalf of agent $i$ at the NE. We have the following lemma on effort profile $\hat{E}$.

Lemma 3.1. Assume that game $G_{r p}$ has at least one Nash equilibrium. The effort profile $\hat{E}$ at the $N E$ satisfies the following system of equations,

$$
\left(2 B-X-X^{T}\right) \cdot\left[\begin{array}{c}
\hat{E}_{1} \\
\vdots \\
\hat{E}_{n}
\end{array}\right]=\boldsymbol{a} .
$$

Proof. As effort profile $\hat{E}$ is the fixed point of the best response mapping, we have,

$$
\begin{aligned}
& \hat{e}_{i i}=\frac{a_{i}}{2 b_{i}}+\frac{\sum_{k=1}^{n} x_{i k} \cdot \hat{E}_{k}}{2 b_{i}} \\
& \hat{e}_{j i}=\frac{x_{j i} \cdot \hat{E}_{j}}{2 b_{i}} \forall j \neq i \Longrightarrow
\end{aligned}
$$

by adding above equations:

$$
\begin{aligned}
2 b_{i} \cdot \hat{E}_{i} & =a_{i}+\sum_{j=1}^{n}\left(x_{i j}+x_{j i}\right) \hat{E}_{j} \forall i \in \mathcal{V} \\
\Longrightarrow \boldsymbol{a} & =\left(2 B-X-X^{T}\right) \cdot\left[\begin{array}{c}
\hat{E}_{1} \\
\vdots \\
\hat{E}_{n}
\end{array}\right]
\end{aligned}
$$

Theorem 3.2. Under Assumption 2, $\left(2 B-X-X^{T}\right)$ is invertible and game $G_{r p}$ has a unique $N E$ given as follows:

$$
\begin{aligned}
{\left[\begin{array}{c}
\hat{E}_{1} \\
\vdots \\
\hat{E}_{n}
\end{array}\right] } & =\left(2 B-X-X^{T}\right)^{-1} \cdot \boldsymbol{a} \\
\hat{e}_{i i} & =\frac{a_{i}}{2 b_{i}}+\frac{\sum_{k=1}^{n} x_{i k} \cdot \hat{E}_{k}}{2 b_{i}} \\
\hat{e}_{i j} & =\frac{x_{i j} \cdot \hat{E}_{i}}{2 b_{j}}, \forall j \neq i
\end{aligned}
$$

Proof. Similar to the proof of Theorem 2.1, we can show that if $2 b_{i} \geq \sum_{j=1}^{n} x_{i j}+x_{j i}, \forall i$, then all eigenvalues of matrix $\left(2 B-X-X^{T}\right)$ are non-zero. Therefore, matrix $\left(2 B-X-X^{T}\right)$ is invertible. By Corollary 2.2, all entries of $\left(2 B-X-X^{T}\right)^{-1}$ are non-negative and $\left[\hat{E}_{1} \cdots \hat{E}_{n}\right]^{T}=\left(2 B-X-X^{T}\right)^{-1} \cdot \boldsymbol{a}$ is a non-negative vector Moreover, by the best response mapping provided in (8), we know that $\hat{e}_{i j}$ can be calculated by the following,

$$
\begin{aligned}
& \hat{e}_{i i}=\frac{a_{i}}{2 b_{i}}+\frac{\sum_{k=1}^{n} x_{i k} \cdot \hat{E}_{k}}{2 b_{i}} \geq 0 \\
& \hat{e}_{i j}=\frac{x_{i j} \cdot \hat{E}_{i}}{2 b_{j}} \geq 0, \forall j \neq i
\end{aligned}
$$

Therefore, the fixed point of the best response mapping is nonnegative and unique, implying the NE of game $G_{r p}$ is unique and can be found by (10).

Remark: It is worth pointing out that for the same weight matrix $X$, the total effort exerted by each agent, $\left[\hat{E}_{1}, \hat{E}_{2}, \cdots, \hat{E}_{n}\right]$, at the NE of the RP-IDS game $G_{r p}$ is the same as the socially optimal effort of the IDS game $G$. That is,

$$
\left[\begin{array}{c}
\hat{E}_{1} \\
\vdots \\
\hat{E}_{n}
\end{array}\right]=\left(2 B-X-X^{T}\right)^{-1} \cdot \boldsymbol{a}=\boldsymbol{e}^{*} \underbrace{\geq}_{\text {By Theorem } 2.3} \hat{\boldsymbol{e}} .
$$

In other words, the introduction of resource pooling incentivizes agents to boost their effort to the socially optimal levels for game $G$. Note that the game $G_{r p}$ has its own socially optimal solution as we discuss in Section 4.3.

Next we show that every agent at the NE of game $G_{r p}$ obtains a higher utility than that attained at the NE of game $G$, i.e., resource pooling improves the utility for all agents.

Theorem 3.3. Let $\hat{E}=\left[\hat{e}_{i j}\right]_{n \times n}$ be the $N E$ of $G_{r p}$ and $\hat{\boldsymbol{e}}$ be the effort profile at the NE of game G. Under Assumption 2, We have:

$$
v_{i}(\hat{E}) \geq u_{i}(\hat{\boldsymbol{e}}), \quad \forall i \in \mathcal{V} .
$$

Proof. Let $\tilde{\boldsymbol{e}}_{i}$ be a vector with length $n$ and all its elements are zero except entry $i$ which is equal to $\hat{e}_{i}$ (effort level of agent $i$ at NE of game $G$ ). By definition of Nash equilibrium we have,

$$
v_{i}(\hat{E}) \geq v_{i}\left(\tilde{\boldsymbol{e}}_{i}, \hat{\boldsymbol{e}}_{-i}\right) \text {. }
$$

As $\hat{E}_{i} \geq \hat{e}_{i}, \forall i$, by (10) and (3) we have $\hat{e}_{i i} \geq \hat{e}_{i}$. Moreover,

$$
\begin{array}{r}
v_{i}\left(\tilde{\boldsymbol{e}}_{i}, \hat{\boldsymbol{e}}_{-i}\right)=-l_{i}+a_{i} \cdot \hat{e}_{i}+a_{i} \sum_{k \neq i}^{n} \hat{e}_{k i}-b_{i} \cdot\left(\hat{e}_{i}\right)^{2} \\
+\left(\hat{e}_{i}+\sum_{k \neq i}^{n} \hat{e}_{k i}\right) \cdot \sum_{j=1}^{n}\left(x_{i j} \cdot\left(\sum_{k \neq i} \hat{e}_{k j}\right)\right) \geq \\
-l_{i}+a_{i} \cdot \hat{e}_{i}-b_{i} \cdot\left(\hat{e}_{i}\right)^{2}+\hat{e}_{i} \cdot \sum_{j=1}^{n} x_{i j} \cdot \hat{e}_{j}=u_{i}\left(\hat{e}_{i}, \hat{e}_{-i}\right)
\end{array}
$$

By (14) and (15), $v_{i}(\hat{E}) \geq u_{i}(\hat{\boldsymbol{e}}) \forall i \in V$.

The following theorem shows that social welfare at the NE of game $G_{r p}$ is higher than the maximum social welfare of game $G$, even though the total effort exerted by each agent is the same under both as noted earlier.

THEOREM 3.4. Let $\hat{E}$ be the effort profile at the NE of game $G_{r p}$ and $\boldsymbol{e}^{*}$ be the socially optimal effort profile in game G. Under Assumption 2 we have,

$$
\sum_{i=1}^{n} v_{i}(\hat{E}) \geq \sum_{i=1}^{n} u_{i}\left(\boldsymbol{e}^{*}\right) .
$$


Proof.

$\sum_{i=1}^{n} v_{i}(\hat{E})=\sum_{i=1}^{n}\left(-l_{i}+a_{i} \hat{E}_{i}-b_{i} \cdot\left[\sum_{j=1}^{n} \hat{e}_{j i}^{2}\right]+\hat{E}_{i} \cdot\left[\sum_{j=1}^{n} x_{i j} \cdot \hat{E}_{j}\right]\right)$

By (12), $\left(e_{i}^{*}\right)^{2}=\hat{E}_{i}^{2}=\left(\sum_{j=1}^{n} \hat{e}_{j i}\right)^{2} \geq \sum_{j=1}^{n}\left(\hat{e}_{j i}\right)^{2}$, and $\hat{E}_{i}=e_{i}^{*}$. Therefore,

$$
\begin{aligned}
\sum_{i=1}^{n} v_{i}(\hat{E}) & \geq \sum_{i=1}^{n}\left(-l_{i}+a_{i} \hat{E}_{i}-b_{i} \cdot \hat{E}_{i}^{2}+\hat{E}_{i} \cdot\left[\sum_{j=1}^{n} x_{i j} \cdot \hat{E}_{j}\right]\right) \\
& =\sum_{i=1}^{n} u_{i}\left(\boldsymbol{e}^{*}\right)
\end{aligned}
$$

We conclude this section by highlighting the role of resource pooling in the IDS game.

1) At the NE, with resource pooling (game $G_{r p}$ ) agents exert higher effort (for themselves and for others) and experience higher utility than the case without resource pooling (game $G$ ); i.e., $\hat{E}_{i} \geq \hat{e}_{i}$, and $v_{i}(\hat{E}) \geq u_{i}(\hat{\boldsymbol{e}})$.

2) Resource pooling induces agents to exert socially optimal levels of effort (under game $G$ ), while improving the social welfare as it allows more judicious spreading of efforts; e.g., $\hat{E}=\boldsymbol{e}^{*}$ and $\sum_{i=1}^{n} v_{i}(\hat{E}) \geq \sum_{i=1}^{n} u_{i}\left(\boldsymbol{e}^{*}\right)$.

\section{DISCUSSION}

\subsection{On Assumption $2 b_{i}>\sum_{j=1}^{n} x_{i j}$}

Throughout the analysis we have used the following assumptions:

- Existence and uniqueness of $\mathrm{NE}$ for game $G$ : $2 b_{i}>\sum_{j=1}^{n} x_{i j}, \forall i$

- Existence and uniqueness of socially optimal strategy profile in game $G$ : $2 b_{i}>\sum_{j=1}^{n} x_{i j}+x_{j i}, \forall i$

- Existence and uniqueness of NE profile in game $G_{r p}: 2 b_{i}>$ $\sum_{j=1}^{n} x_{i j}+x_{j i}, \forall i$

The reason behind these assumptions is to prevent the model from becoming pathological: if the cost of effort is sufficiently low, then there may not exist NE or socially optimal strategy, and it may be beneficial for the agents to exert very high effort with unbounded utility.

Example 4.1. Consider a network with $x_{i i}=0, x_{i j}=\frac{1}{n-1} \forall i, j \in$ $V, i \neq j$ and $b_{i}=1$. Under these parameters Assumption 2 does not hold. Moreover, set $e_{i}=r, \forall i \in V$. We have:

$$
\begin{aligned}
\sum_{i=1}^{n} u_{i}(\boldsymbol{e}) & =\sum_{i=1}^{n}\left(-l_{i}+(r) a_{i}-b_{i} \cdot r^{2}+r^{2} \sum_{j=1}^{n} x_{i j}\right) \\
& =\left(-\sum_{i=1}^{n} l_{i}\right)+r \cdot\left(\sum_{i=1}^{n} a_{i}\right),
\end{aligned}
$$

which is a linear function in $r$ and is unbounded. In this case the socially optimal effort does not exist.

\subsection{Voluntary Participation in RP}

As investment in security is a non-excludable public good, an agent can benefit even if it chooses not to participate in an incentive mechanism. As a result, designing a mechanism which incentivizes the agents to voluntarily participate and exert socially optimal effort levels is not straightforward. In [20] it was shown that no taxation mechanism is able to implement the socially optimal solution while guaranteeing both weak budget balance and voluntary participation. For this reason, it is important to check whether agents will voluntarily participate in resource pooling. In what follows, we first define this notion and then show that under resource pooling the voluntary participation property is satisfied.

Definition 4.2 (Voluntary Participation (VP)). Consider game $G_{r p}^{k}$ where agent $k$ opts out of RP and only invests in himself and nobody else invest in agent $k\left(e_{k j}=e_{j k}=0, \forall j \neq k\right)$, while other agents participate in RP. Let $\stackrel{\circ}{E}=\left[\stackrel{\circ}{e}_{i j}\right]_{n \times n}$ be the NE of game $G_{r p}^{k}$ and $v_{i}(\stackrel{\circ}{E})$ be the utility of agent $i$ at the NE. We say that resource pooling has the voluntary participation property with respect to agent $k$, if

$$
v_{k}(\stackrel{\circ}{E}) \leq v_{k}(\hat{E}),
$$

where $\hat{E}$ is the effort profile at the NE of game $G_{r p} \cdot{ }^{3}$ If the above is true for all $k \in \mathcal{V}$, then we say that resource pooling has the voluntary participation property.

By the definition of NE, effort profile $\hat{E}=\left[\hat{e}_{i j}\right]_{n \times n}$ satisfies the following,

$$
v_{i}\left(\hat{\boldsymbol{e}}_{i}, \hat{\boldsymbol{e}}_{-i}\right) \geq v_{i}\left(\boldsymbol{e}_{i}, \hat{\boldsymbol{e}}_{-i}\right) \forall \boldsymbol{e}_{i} \in R^{n},
$$

where $\hat{\boldsymbol{e}}_{i}=\left[\hat{e}_{i 1}, \cdots, \hat{e}_{i n}\right]$. It is worth noting that the above definition does not imply VP defined in (17). The following theorem suggests that resource pooling always satisfies the VP property.

Theorem 4.3. If Assumption 2 holds, then agent $i$ achieves higher utility at the NE of game $G_{r p}$, than his utility at the NE of game $G_{r p}^{i}$ for all $i \in \mathcal{V}$. That is, resource pooling always satisfies the VP property.

Proof. See Appendix.

As no one has incentive to deviate from resource pooling unilaterally, resource pooling is a better way to improve social welfare as compared to taxation mechanisms which are not able to satisfy the voluntary participation and budget balance constraint simultaneously [20].

\subsection{On the Socially Optimal Outcome of Game $G_{r p}$}

While the NE of the RP-IDS game $G_{r p}$ achieves socially optimal levels of effort defined for the IDS game $G$, the introduction of resource pooling means that each agent now has a bigger action space, thereby giving rise to a different social optimum for this new game. We next show how this new optimum can be computed. Let $E^{*}=\left[e_{i j}^{*}\right]_{n \times n}$ be the socially optimal effort profile for the RP-IDS game:

$$
\begin{aligned}
E^{*} & =\arg \max _{E \in R_{+}^{n \times n}} \sum_{i=1}^{n} v_{i}(E) \\
& =\arg \max _{E \in R_{+}^{n \times n}} \sum_{i=1}^{n}\left[-l_{i}+a_{i} E_{i}-b_{i} \cdot\left(\sum_{j=1}^{n} e_{j i}^{2}\right)+E_{i} \sum_{j=1}^{n} x_{i j} E_{j}\right] .
\end{aligned}
$$

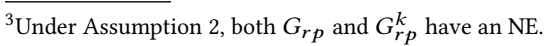


The assumption below ensures the existence of a solution.

Assumption 3. $2 b_{i}>n \cdot \sum_{j=1}^{n}\left(x_{i j}+x_{j i}\right), \forall i \in \mathcal{V}$

Under Assumption 3, it is easy to check that $g(E)=\sum_{i=1}^{n} v_{i}(E)$ is strictly concave in $E$. By the first order condition, $E^{*}$ satisfies the following:

$$
\begin{aligned}
& \left.\frac{\partial g(E)}{\partial e_{i i}}\right|_{E=E^{*}}=a_{i}-2 b_{i} e_{i i}^{*}+\sum_{j=1}^{n}\left(x_{i j}+x_{j i}\right) \cdot E_{j}^{*}=0 \\
& \left.\frac{\partial g(E)}{\partial e_{k i}}\right|_{E=E^{*}}=a_{i}-2 b_{i} e_{k i}^{*}+\sum_{j=1}^{n}\left(x_{i j}+x_{j i}\right) \cdot E_{j}^{*}=0 \\
\Longrightarrow & n \cdot a_{i}-2 b_{i} E_{i}^{*}+n \cdot \sum_{j=1}^{n}\left(x_{i j}+x_{j i}\right) \cdot E_{j}^{*}=0, \forall i \in \mathcal{V}, \\
\Longrightarrow & \left(2 B-n \cdot\left(X+X^{T}\right)\right) \cdot\left[\begin{array}{c}
E_{1}^{*} \\
\vdots \\
E_{n}^{*}
\end{array}\right]=n \cdot \boldsymbol{a} .
\end{aligned}
$$

Similar as before, we can show that under Assumption 3, $(2 B-n$. $\left.\left(X+X^{T}\right)\right)$ is invertible. Thus the optimal outcome $E^{*}$ is given by:

$$
\begin{aligned}
{\left[\begin{array}{c}
E_{1}^{*} \\
\vdots \\
E_{n}^{*}
\end{array}\right]=n \cdot\left(2 B-n \cdot\left(X+X^{T}\right)\right)^{-1} \cdot \boldsymbol{a} } \\
e_{k i}^{*}=\frac{a_{i}}{2 b_{i}}+\frac{\sum_{j=1}^{n}\left(x_{i j}+x_{j i}\right) \cdot E_{j}^{*}}{2 b_{i}}, \forall i, k \in \mathcal{V}
\end{aligned}
$$

By Corollary 2.2, $\left(2 B-n \cdot\left(X+X^{T}\right)\right)^{-1} \cdot \boldsymbol{a} \geq\left(2 B-\left(X+X^{T}\right)\right)^{-1} \cdot \boldsymbol{a}$ which implies that $E_{i}^{*} \geq \hat{E}_{i}, \forall i$, i.e., the total effort exerted on behalf of agent $i$ improves under the social optimum compared to that under the NE of game $G_{r p}$. As before, not all agents may attain higher individual utility under $E^{*}$ as compared to their utility under NE $\hat{E}$. Examples are provided in the Appendix.

\section{CONCLUSIONS}

We considered an IDS game with positive externality, and introduced a resource pooling augmented IDS game, the RP-IDS game, to examine the effect of using resource pooling as a mechanism to incentivize higher effort levels by interdependent agents. We showed that (1) resource pooling increases the total effort exerted on behalf of each agent as compere to no resource pooling, (2) each agent experiences higher utility under resource pooling as compared to no resource pooling, (3) social welfare at the NE of the RP-IDS game is higher than the optimal social welfare under the IDS game, and (4) agents voluntarily participate in resource pooling. An interesting future direction is to consider the case where agents only pool their resources within an alliance or coalition.

Appendix: Proofs are given in [1].

\section{REFERENCES}

[1] Online Appendix. Available at https://www.dropbox.com/sh/d1w9349qpqby2xu/ AAAa-AGbKhZSWDJk1dmkR5ESa?dl=0.

[2] Saurabh Amin, Galina A. Schwartz, and S. Shankar Sastry. 2013. Security of interdependent and identical networked control systems. Automatica 49, 1 (2013), 186 - 192. https://doi.org/10.1016/j.automatica.2012.09.007

[3] Ozan Candogan, Kostas Bimpikis, and Asuman Ozdaglar. 2012. Optimal pricing in networks with externalities. Operations Research 60, 4 (2012), 883-905.
[4] Juntao Chen and Quanyan Zhu. 2019. Interdependent Strategic Security Risk Management with Bounded Rationality in the Internet of Things. IEEE Transactions on Information Forensics and Security (2019).

[5] Joan de Marti and Yves Zenou. 2015. Network games with incomplete information. Journal of Mathematical Economics 61 (2015), 221 - 240. https://doi.org/10.1016/j. jmateco.2015.10.002

[6] Jens Grossklags, Nicolas Christin, and John Chuang. 2008. Secure or insure?: a game-theoretic analysis of information security games. In Proceedings of the 17th international conference on World Wide Web. ACM, 209-218.

[7] Jens Grossklags, Svetlana Radosavac, Alvaro A. Cárdenas, and John Chuang. 2010. Nudge: Intermediaries' Role in Interdependent Network Security. In Trust and Trustworthy Computing, Alessandro Acquisti, Sean W. Smith, and Ahmad-Reza Sadeghi (Eds.). Springer Berlin Heidelberg, Berlin, Heidelberg, 323-336.

[8] Nasimeh Heydaribeni and Achilleas Anastasopoulos. 2018. Distributed Mechanism Design for Multicast Transmission. In 2018 IEEE Conference on Decision and Control (CDC). IEEE, 4200-4205.

[9] Nasimeh Heydaribeni and Achilleas Anastasopoulos. 2019. Distributed Mechanism Design for Network Resource Allocation Problems. arXiv preprint arXiv:1904.01222 (2019)

[10] A. R. Hota and S. Sundaram. 2018. Interdependent Security Games on Networks Under Behavioral Probability Weighting. IEEE Transactions on Control of Network Systems 5, 1 (March 2018), 262-273. https://doi.org/10.1109/TCNS.2016.2600484

[11] Christos Ioannidis, David Pym, and Julian Williams. 2016. Is Public Co-Ordination of Investment in Information Security Desirable? Journal of Information Security 7, 2 (2016), 60-80.

[12] Libin Jiang, Venkat Anantharam, and Jean Walrand. 2011. How bad are selfish investments in network security? IEEE/ACM Transactions on Networking (TON) 19, 2 (2011), 549-560.

[13] Mohammad Mahdi Khalili, Parinaz Naghizadeh, and Mingyan Liu. 2017. Designing cyber insurance policies in the presence of security interdependence. In Proceedings of the 12th Workshop on the Economics of Networks, Systems and Computation. ACM, 7.

[14] Mohammad Mahdi Khalili, Parinaz Naghizadeh, and Mingyan Liu. 2017. Designing cyber insurance policies: Mitigating moral hazard through security prescreening. In International Conference on Game Theory for Networks. Springer, 63-73.

[15] Mohammad Mahdi Khalili, Parinaz Naghizadeh, and Mingyan Liu. 2018. Designing cyber insurance policies: The role of pre-screening and security interdependence. IEEE Transactions on Information Forensics and Security 13, 9 (2018), 2226-2239.

[16] Mohammad Mahdi Khalili, Xueru Zhang, and Mingyan Liu. 2019. Public Good Provision Games on Networks with Resource Pooling. In Network Games, Control, and Optimization. Springer, 271-287.

[17] R. J. La. 2017. Effects of Degree Correlations in Interdependent Security: Good or Bad? IEEE/ACM Transactions on Networking 25, 4 (Aug 2017), 2484-2497. https://doi.org/10.1109/TNET.2017.2691605

[18] Aron Laszka, Mark Felegyhazi, and Levente Buttyan. 2015. A survey of interdependent information security games. ACM Computing Surveys (CSUR) 47, 2 (2015), 23

[19] R Ann Miura-Ko, Benjamin Yolken, John Mitchell, and Nicholas Bambos. 2008. Security decision-making among interdependent organizations. In Computer Security Foundations Symposium, 2008. CSF'08. IEEE 21st. IEEE, 66-80.

[20] Parinaz Naghizadeh and Mingyan Liu. 2016. Exit equilibrium: Towards understanding voluntary participation in security games. In Computer Communications, IEEE INFOCOM 2016-The 35th Annual IEEE International Conference on. IEEE, 1-9.

[21] Ranjan Pal, Leana Golubchik, Konstantinos Psounis, and Pan Hui. 2017. Security pricing as enabler of cyber-insurance a first look at differentiated pricing markets. IEEE Transactions on Dependable and Secure Computing (2017).

[22] Walid Saad, Tansu Alpcan, Tamer Basar, and Are Hjorungnes. 2010. Coalitional game theory for security risk management. In 2010 Fifth International Conference on Internet Monitoring and Protection. IEEE, 35-40.

[23] Abhinav Sinha and Achilleas Anastasopoulos. 2017. Distributed mechanism design with learning guarantees. In 2017 IEEE 56th Annual Conference on Decision and Control (CDC). IEEE, 5000-5007.

[24] Iman Vakilinia and Shamik Sengupta. 2017. A coalitional game theory approach for cybersecurity information sharing. In MILCOM 2017 - 2017 IEEE Military Communications Conference (MILCOM). 237-242. https://doi.org/10.1109/MILCOM. 2017.8170845

[25] Iman Vakilinia and Shamik Sengupta. 2019. A Coalitional Cyber-Insurance Framework for a Common Platform. IEEE Transactions on Information Forensics and Security 14, 6 (June 2019), 1526-1538. https://doi.org/10.1109/TIFS.2018. 2881694

[26] Iman Vakilinia and Shamik Sengupta. 2019. Fair and Private Rewarding in a Coalitional Game of Cybersecurity Information Sharing. IET Information Security (2019).

[27] Hal Varian. 2004. System Reliability and Free Riding. Springer US, Boston, MA, 1-15. https://doi.org/10.1007/1-4020-8090-5_1 